\title{
From Immersion to Addiction in Videogames
}

\author{
May-li Seah \\ University College London \\ 31-32 Alfred Place \\ London WC1E 7DP, UK
}

mayli.seah@gmail.com

\author{
Paul Cairns \\ University of York \\ Heslington \\ York YO10 5DD, UK \\ +441904 434751 \\ pcairns@cs.york.ac.uk
}

\begin{abstract}
Immersion is commonly described by gamers and gamereviewers as an important aspect of a videogame. In this paper, we investigate the relationship between the immersive experience of videogames and the addictive nature of games. Building on Charlton's (2002) study of addiction and engagement in computing, we conducted a questionnaire study of people who play videogames. It seems that videogames blur the distinction between addiction and high engagement even more than generic computing. In a follow up diary study, the degree of immersion whilst playing was found to be strongly correlated $(\mathrm{r}=0.763)$ with the addiction/engagement score. Overall, these studies suggest that the degree of immersive experience is closely related to how addictive or engaging people find videogames and moreover that addiction seems to be an extreme form of engagement and immersion.
\end{abstract}

\section{Categories and Subject Descriptors}

J.4 [Social and behavioral sciences] Psychology; K.8.0 [Personal Computing]: General - games.

\section{General Terms}

Measurement, Experimentation, Human Factors

\section{Keywords}

Videogames, immersion, addiction, engagement

\section{IMMERSION AND ADDICTION}

Immersion is a term commonly used by gamers and gamereviewers as an important element of videogames [5]. It is colloquially understood as the sense of being lost in the game where players lose awareness of their surroundings and their day-to-day concerns [16]. Whilst immersion is generally understood to be a positive element of the experience, it is widely held that becoming too absorbed in videogames can be unhealthy and lead to addiction. Indeed, addiction clinics are being established to deal with videogames [4] and in extreme cases, people have died because of obsessively playing games [3]. This paper therefore considers the immersive experience of playing videogames in relation to addiction.

Naively, it seems that the more a player becomes immersed in a

\footnotetext{
(c) The Author 2008.
}

Published by the British Computer Society game, the more likely this is to lead to addiction. However, as will be discussed in the next section, defining addiction in games and computing more generally is not straightforward because of the lack of a strongly destructive element to such an addiction, unlike other addictions to things like alcohol or gambling [9]. We will also briefly review what we mean by immersion in games to distinguish it from other notions of positive experience such as flow [13] or Presence [19].

The first study reported here is intended to build on existing work on addiction to computing and apply it to addiction to videogames specifically. Using an adapted form of Charlton's [10] questionnaire, factor analysis suggests that the distinction between engagement in addiction is somewhat artificial for games. Interestingly, since conducting this research, Charlton and Danforth have also investigated addiction in games [11]. Our results provide a possible reinterpretation of these later findings further blurring the distinction between addiction and engagement in videogames.

Addiction and engagement in these terms are attitudes to the general experience of playing. Immersion however is intended to address the actual experience of specific instances of play. Or more succinctly, when talking about individual players, addiction and engagement are traits whereas immersion is a state. Thus, in the second study, we look at the relationship between immersion and addiction/engagement in players who play a lot. The findings suggest a strong correlation between how immersed a player feels and the degree to which they are addicted/engaged.

In summary, engagement is hard to distinguish from addictive behaviours around playing videogames. Immersion, the actual experience of playing, does relate to engagement and may be a factor leading to addiction in videogames.

\section{ADDICTION AND ENGAGEMENT}

Addiction in computing is not new with early studies around computers suggesting a strong link between addiction and the behaviour of some programmers [24]. And colloquially there is a lot of talk of being addicted to playing videogames [15]. However, it is also clear that, in describing people addicted to computing activities, it does not necessarily mean that these people are suffering the same socially destructive and pathological behaviours associated with other addictions such as alcohol and drugs. Addicted programmers still produce functioning programs and people addicted to videogames do not generally resort to crime to fund their habit. For this reason, Charlton [9] makes the distinction between addiction and high engagement. Many users of computers generally and videogames specifically exhibit high engagement in the sense of spending substantial amounts of time in these activities and also spending a lot of time outside of these activities thinking about them. A good example of this is in the massively- 
multiplayer online game, World of Warcraft. Within the first 8 months of the game being released, $15 \%$ of the avatars must have been played for the equivalent of two months worth of forty hour weeks in order to achieve level 60 [14]. This certainly indicates a high level of engagement in up to 20,000 people (it is hard to be sure because players may have more than one avatar). However, given the lack of media furore, it seems reasonable that these 20,000 or so people remained reasonably well adjusted members of society, that is, they did not behave like other sorts of addicts.

This is not to say that videogames do not have a negative impact, akin to addiction. Chappell et al. [8] showed that there are many online forums where people have spoken about the negative impact that extreme gaming has had on their lives.

A common tool for diagnosing addiction, amongst other mental disorders is the DSM, currently in its fourth version, DSM-IV [2]. However, the DSM-IV no longer refers explicitly to addiction but rather to dependence, emphasizing the notion of ingesting substances as part of the addiction. Brown [6] however argued that behaviours themselves could be addictive. To this end he produced a checklist of attributes for behavioural addiction. Like many DSM diagnostic methods, it is enough for a person to satisfy all or some set number of the attributes to be defined as addicted.

Charlton [9] however felt that in computing, it was not clear that behavioural addiction would be sufficiently distinct from high engagement and Brown's checklist would be a misleading indicator of addiction. His survey study of 404 undergraduates therefore aimed to position ten criteria based on Brown's behavioural addiction attributes in relation to the Computer Apathy and Anxiety Scale (CAAS) that he had previously developed [10]. He found that the CAAS produced two separate factors corresponding to engagement and addiction (as well as a further comfort factor) but that Brown's criteria split between these two factors. This suggested that criteria related to the engagement factor are more peripheral than those criteria relating to addiction. These latter criteria, Charlton deemed to be core criteria for computing addiction. Charlton also proposed a developmental model by which high engagement exhibiting the peripheral behaviours leads to addiction and behaviours meeting the core criteria.

Thus, it seemed reasonable that Charlton's adapted CAAS could be used in this work to make a suitable measure of addiction in videogames. However, as Charlton had studied computing in general rather than videogames in particular, it is important to repeat Charlton's study in order to examine its validity in the more restricted domain. In addition, we included Brown's criteria of behavioural addiction to help define the factor structure that might emerge and in the hope that they would similarly split into core and peripheral criteria of addiction to gaming.

Since conducting our work, Charlton and Danforth reported investigating the same question in relation to videogames [11]. They asked questions from the CAAS but adapted them to ask about a specific game, Asheron's Call [22], and only asked questions that had loaded on the engagement and addiction factors in the previous study. They found again the division between engagement and addiction and also that Brown's criteria split again into core and peripheral attributes.

What is surprising is that we did not find such a clear division of factors between engagement and addiction in videogames. We therefore report our investigation using the CAAS and, from that, present a possible re-interpretation of Charlton and Danforth's findings.

\section{IMMERSION IN GAMES}

Immersion is generally understood as the experience of being "lost" in a particular experience. Whilst commonly applied as an important feature of videogames, it is not fully clear what is actually meant by immersion though it is possible to have a graded experience of immersion. That is, it is possible to be more or less immersed in games and that there are fleeting moments of total immersion when a person is entirely lost in the game they are playing [5].

In order to study immersion in more detail, we have devised a questionnaire to measure immersion drawing on the findings of earlier work [5] and related concepts such as flow [13] and cognitive absorption [1]. For instance, there are questions on the sense of challenge, of losing awareness of surroundings and of focus on the game. A larger scale validation of the questionnaire suggests that immersion as measured by the questionnaire is a mix of psychological factors, specifically the cognitive and emotional involvement in the game and the sense of dissociation from the real world, and game factors of the challenge and control provided by the game [16]. Immerison in videogames in this sense is the psychological experience of being involved in a game thanks to the opportunities it offers.

We hold that immersion is distinct from other concepts, such as flow, presence and cognitive absorption. Flow is a harmonious psychological state whereby a person is engaged in an activity that is challenging but not beyond the skills of the person and has a clear sense of progression towards a goal. The outcome is a positive and rewarding experience. Games can fail to provide flow whilst still being immersive. Many games do not provide clear goals leaving it to the player to either work out what is going on and what to do, for example in Myst IV [23], or to set their own goals, as in Grand Theft Auto 3 [18]. Playing can also be very frustrating and far from positive because of an inability to overcome a particular obstacle without necessarily being less immersive. Conversely, even when failing to achieve a clear, well laid-out objective (for example, killing the boss monster), people can have a satisfying and immersive experience. Thus whilst immersion need not be coincident with flow, it does seem that immersion is a precursor of flow. During flow, people are wholly engaged in their activity to the exclusion of all other concerns. This sense of being "lost to the world" matches well with the colloquial sense of immersion.

Indeed, GameFlow [20] makes immersion an explicit part of the enjoyment of playing games as GameFlow is held to lead to flow experiences whilst playing. Immersion, as defined here, overlaps with three of the factors of GameFlow, namely concentration (which equates to cognitive involvement), challenge, control and immersion (emotional involvement, real world dissociation). This supports the understanding that immersion is an important aspect of a gaming experience. However, GameFlow also includes other factors such as player skills, clear goals, feedback and social interaction. These set GameFlow more in line with flow whereas immersion, we hold, is set apart from flow because of the superfluity of these same factors in having an immersive experience.

Immersion is also distinct from the notion of presence [19,25]. Presence is the sense of being present in some virtual world as opposed to the real world. It is clear that people who feel as if they are in some virtual world are lost to this one and in that sense are immersed. Immersion in this sense though is one of perceptual immersion as opposed to psychological immersion 
[8]. These two types of immersion dissociate. For example, many games, such as Tetris, do not offer the players the opportunity to feel presence, as there is no virtual environment in which to be present, yet can provide very immersive experiences. Also, it is possible to imagine doing a tedious task in a virtual environment so that time drags and the player is not challenges thus not achieving any sense of psychological immersion. It is the psychological experience of immersion that we focus on here.

Cognitive absorption [1] is intended to capture people's propensity to become absorbed in the activities around using a computer. In this sense, cognitive absorption is like Charlton's notion of engagement and indeed both may seem to have overlap with immersion. However, in using the immersion questionnaire, people are asked to rate specific experiences of playing videogames. Cognitive absorption and CAAS instead ask about people's general disposition towards computing activities. Cognitive absorption though does consider the subjective experiences of people and is concerned with the enjoyment, challenge and focus of people using computers. In this sense, immersion overlaps with a notional state of being cognitively absorbed rather than the disposition of cognitive absorption. With CAAS however, despite the apparent similarity between the terms of high engagement and immersion, the CAAS questions refer more to attitudes to using computers and people's behaviour around using computers. The immersion questionnaire focuses instead on people's subjective, internal state as a result of playing a game. As a result there is only one question in the CAAS that directly maps to a question in the immersion questionnaire about the degree of challenge felt when using computers. All other questions address quite different factors from immersion.

Immersion, then, is related to these other notions but is distinct because of how it occurs and in that it addresses specific experiences of playing. This work aims to position immersion in relation to addiction to see how the actual experience of playing a videogame influences a person's attitude, their perception of addiction, towards playing videogames.

\section{STUDY 1: SPECIFYING ADDICTION}

The purpose of this study was to replicate Charlton's study [9] in order to see if his adapted CAAS provided a suitable instrument for measuring addiction in videogames. Exploratory factor analysis was used to analyse the results of the questionnaire since we were considering a modified version of Charlton's questionnaire in a specialized context rather than confirming the previous work [17].

\subsection{Participants}

Participants were mostly students across a wide range of subjects. There were 85 participants, 48 men and 37 women. The average age was 20.9 years $(\mathrm{SD}=1.84$ years). This would be a small sample for exploratory factor analysis but as the basic factor structure of the questionnaire is already understood albeit in a different context, this is a large enough sample meeting Kline's guidelines of there being a ratio of around 2:1 of participants to questions and of around 20:1 of participants to factors [17].

\subsection{Materials}

Charlton's adapted CAAS was the basis for the questionnaire used in this study. However, the statements in it were for the generic experience of computing so in general "computing" was replaced by "computer games" and two questions were dropped entirely as being meaningless in this context, namely "Videogames are too scientific for me" and "I prefer to use pen and paper rather than a word processor...". These statements loaded on the Comfort factor of the CAAS and so were believed not to affect using the CAAS to measure engagement and addiction. Participants were asked to rate each statement in the questionnaire on a five point scale of agreement from strongly disagree to strongly agree. The final statements ended up in a very similar form to those of Charlton's later study except that where we have "videogames", Charlton has used the name of specific game, Asheron's Call [22].

All statistical analyses were done in SPSS v13.0 and have been more recently checked in $\mathrm{v} 15.0$

\subsection{Procedure}

Participants were approached opportunistically to complete the questionnaire. Each person was briefed on the usage of the data in accordance with the ethical clearance provided on the project.

\subsection{Results}

Based on Charlton's study [9], we expected the questionnaire to produce three factors. A principal components analysis (PCA) was performed to check. The unrotated first factor accounted for almost $30 \%$ of the data on its own and indicated a good coherence of the questionnaire in this context as all but seven questions strongly loaded on the first factor. The scree plot suggested either a three or four factor solution so we went on to do further analysis on both solutions. Principal axis factoring was used to perform the factor analysis with direct oblimin rotation of factors. These are the same methods used by Charlton. However, Charlton analysed the pattern matrix to define his factors whereas, because we found modest correlations between the rotated factors, we used the structure matrix as it provides a more accurate picture of the statistical relationship between factors and variables [17]. Correlations between variables and factors of more than 0.35 were taken to indicate that the variable loads on the factor. This is slightly higher than Charlton's 0.32 but is slightly more conservative to account for our small sample.

The three factor model accounted for $42.8 \%$ of the variance in the data and the four factor model for $46.6 \%$. When it came to interpreting the two models, the three factor model presented a clearer division of the factors across questions and lower correlations between the factors. This makes the factors easier to interpret and so is presented here. Table 1 summarises the questions and their loadings on each of the three rotated factors. The factor structure for Charlton's original study is included in the table for comparison purposes. Where a question loads on more than one factor, it is assigned to the factor with the higher loading. Reading down the table then, the rows are grouped into their corresponding factors with the last four questions belonging to no particular factor. Within the factor groupings, the rows are also grouped to help reveal Charlton's original factor structure.

The most striking contrast with Charlton's factor structure is that whereas he found two factors, one corresponding to engagement and another to addiction, we have one large factor covering both engagement and addiction. Indeed, it includes all but one of Brown's criteria for behavioural addiction. Even then, question 28 is close to loading on Factor 1. This strongly suggests that in videogames, the distinction between engagement and addiction is much less clear cut. 


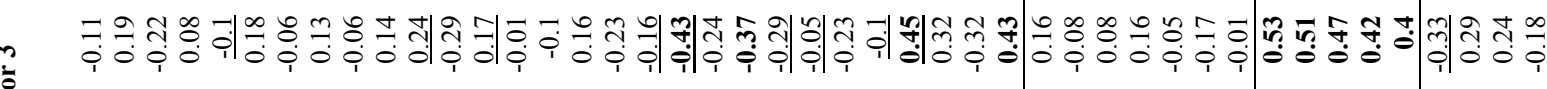

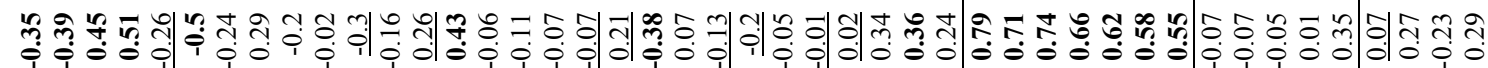

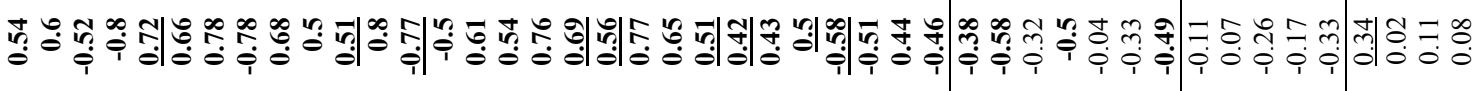

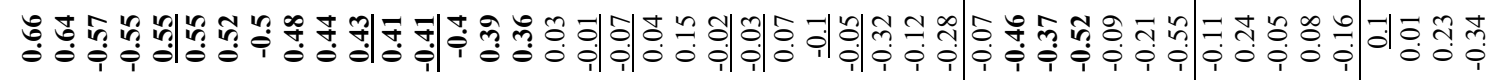
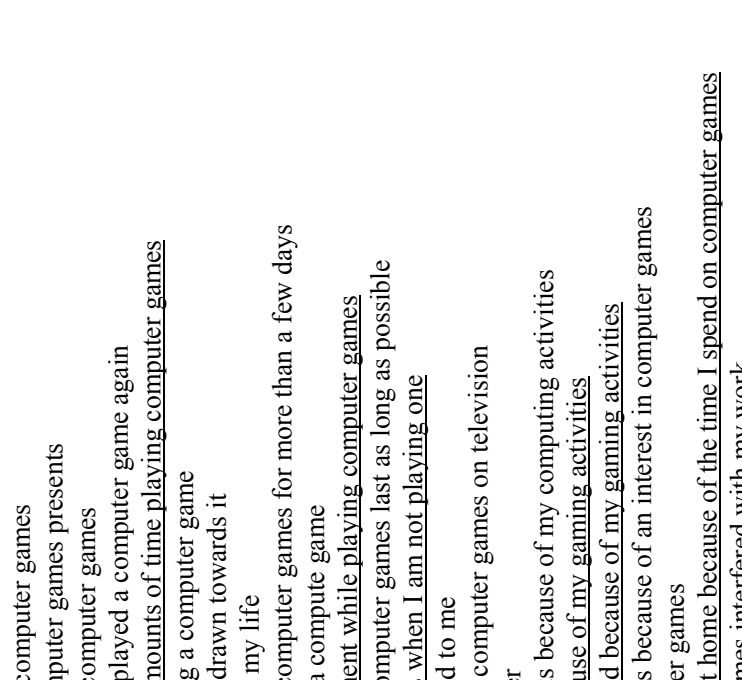
Factors 2 and 3 overlap substantially with Charlton's Comfort factor but divide it up into two roughly equal parts. Factor 2 can be reasonably interpreted as Attitude to videogames and whether people have an underlying interest in them. This becomes clearer when also taking into consideration questions that crossload on both Factors 1 and 2. The correlation between factors 1 and 2 is $r=-0.223$ which is small but is quite high for factor analysis. This suggests that Attitude has some bearing on engagement/addiction. Factor 3 can be reasonably interpreted as Confidence in playing videogames.

This partitioning of Comfort in the context of videogames could be understood as due to the degree to which people have to use computers. Where people have to use computers, say in a work context, their attitude to using computers and their confidence in using them are intertwined through necessity. By contrast, in videogames, people normally make the choice to play videogames but having chosen (or not), they may have a varying degree of confidence in their ability to play.

The four questions that are not incorporated into these factors are worth discussing. Question 28 was already discussed and it is close to loading on either Factor 1 or Factor 3. Whilst it would make most sense in the context of Factor 1, it is hard to see how it might sensibly be considered an aspect of Confidence. Question 29 may not load because socializing can now take place through online games $[12,14]$ and hence the social aspect of gaming is orthogonal to engagement and addiction. Questions 41 and 45 are about calmness and irritation with games. In the context of general computing, it is clear that these make sense as aspects of Comfort, however many games are not designed to keep the player calm, quite the contrary, and failure to progress can be irritating but not at all demotivating. Thus, it can be seen how calmness and irritation are independent of a disposition to engage with games depending on what different players seek from the playing experience. Of course, that these questions are not accounted for in this factor structure may be due to small sample sizes and larger samples may clarify the picture.

\subsection{Comparison with Charlton and Danforth}

Similar to this study, Charlton and Danforth [11] used their questionnaire to probe for addiction and engagement in the game Asheron's Call [22]. They found a clear two factor structure corresponding to the two factors of engagement and addiction found in general computing. This contrasts with our finding of single factor of engagement/addiction. Moreover, in their study, the ten questions relating to Brown's criteria of behavioural addiction split cleanly with the core criteria loading on the addiction factor and the peripheral criteria loading on the engagement factor. Thus, it would seem there is some disagreement between their factor structure and that described above.

However, in contrast to both their previous work and this study, they used only those questions specifically related to the engagement and addiction factors. Thus, to compare our findings with this study, we performed an identical factor analysis on only those questions used in [11].

A principal component analysis and scree plot also suggested a two factor model for our restricted analysis. The first factor loaded on all but one of the questions (question 29 which was also was not well accounted for in our full analysis). The first factor also accounted for a hefty $39.5 \%$ of the overall variance.
Replicating the analysis in [11], we performed a principal axis factoring with direct oblimin rotation and analysed the pattern matrix. At first glance, it would seem that this produced a very similar two factor structure to [11] with one factor clearly relating to addiction and the other to engagement. There was not a perfect match, though. Specifically, two of Brown's core criteria for behavioural addiction appeared in our engagement factor and not the addiction factor. Looking more closely, there was a very high correlation between the two factors $(\mathrm{r}=0.438)$ which is very unusual in factor analysis and would undermine using the pattern matrix to interpret the factors [17]. In fact, [11] also reports a large correlation between the two factors reported there ( $\mathrm{r}=-0.327$, the negative sign being an artefact of factor analysis). From analysing the structure matrix instead, which reports correlations between factors and variables, it was clear that the two factor structure was somewhat artificial as many questions crossloaded substantially on both factors. This makes it difficult to provide clear and distinctive interpretations of the factors which together with the high correlation between the factors suggests that the two factor model is not well supported by our data.

\subsection{Discussion}

The aim of the study was to see if Charlton's adapted CAAS questionnaire was suitable for measuring addiction in videogames rather than computing in general. The analyses done here suggest that it is an effective tool as it is largely coherent and provides a factor structure that is different from the original study but can be understood in terms of Charlton's original factors. Indeed, Charlton maintains the close link between engagement and addiction and the findings here suggest that in videogames that association is even closer.

A factor analysis just restricted to those elements directly addressing engagement and addiction suggests a possible two factor structure to engagement/addiction. Actually, there is such a close relationship between these factors that making a distinction is somewhat artificial and a single factor model of engagement/addiction provides a more coherent interpretation. Thus, Charlton's CAAS does provide a feassible measure of addiction in videogames as most of the CAAS loads on the addiction/engagement factor.

With regards to Brown's criteria, it is now not clear whether it is meaningful to make the distinction between core and peripheral criteria for addiction in videogames. However, this may complement Charlton's developmental model of addiction. In his analysis, high engagement in computing activities led to addiction. However, that these aspects were split across two factors could also mean that high engagement could vary somewhat independently of addiction, that is, it could be possible to be highly engaged without necessarily being highly addicted and vice versa. However, in the single factor model found here, it would seem that engagement and addiction are on a single sliding scale so that higher engagement does entail more addiction. Thus, high engagement in games could be a reinforcing cycle leading to addiction which need not be the case in other sorts of computing activities.

Of course, these questionnaires probe for disposition of individuals towards videogames. That is, a person scoring highly on the engagement/addiction scale is prone to experience high engagement and exhibit some attributes of behavioural addiction. However, this does not relate the disposition of the player to the actual experience of playing videogames. This is the focus of the next study. 


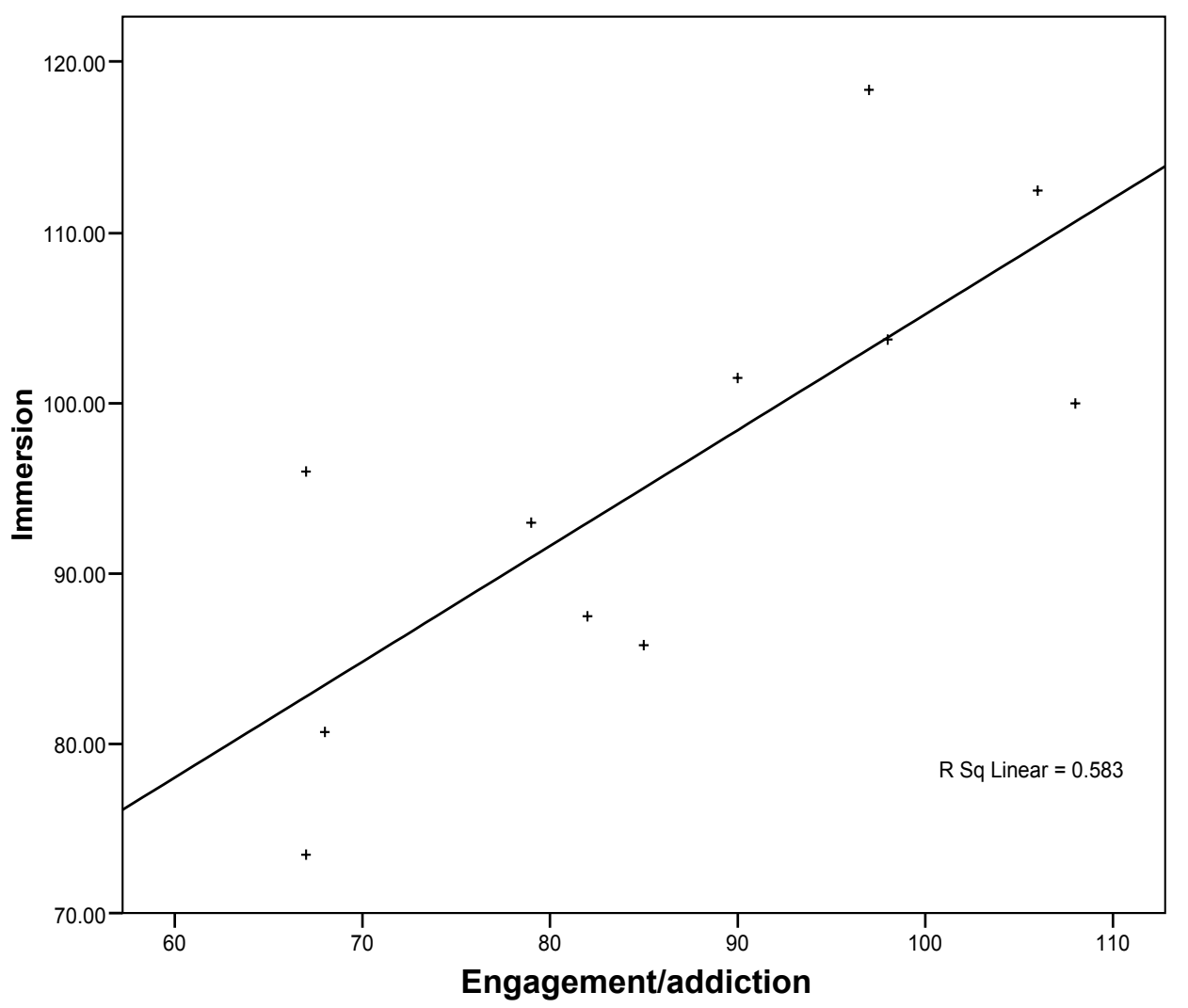

Figure 1. Immersion scores plotted against engagement/addiction scores with linear regression line.

\section{STUDY 2: IMMERSIVE EXPERIENCE AND ADDICTIVE TENDENCY}

Immersion is one aspect of the experience of playing videogames and is widely held to be important to the overall success of a game. The aim of this study is to relate the degree of immersion that players experiences to their tendency to be addicted to playing videogames. It is expected that immersion and addiction/engagement are positively correlated because immersion leads to extended playing sessions due to loss of time and self awareness and this leads to outcomes associated with behavioural addiction.

\subsection{Study design}

The study is a correlational design between immersion score as measured by a previously developed questionnaire and addiction/engagement as measured by Factor 1 of the adapted CAAS. The problem with measuring immersion in videogames is that players have individual preferences and experiences of previous games. In a lab-based study, we have found in previous experiments that player preference for a game strongly influences their ability to be immersed and also unfamiliarity with the game used in a lab may confound the immersive experience. Instead then, a more naturalistic method was used to gather immersion data. Participants were asked to keep a diary of playing sessions and, after longer sessions, to fill out the immersion questionnaire. Over the timescale of the project, this required that participants play regularly and for longer periods. Thus, participants were chosen from the previous study on the basis of how much they reported playing videogames each week.

The hypothesis was that addiction/engagement and immersion correlate positively

\subsection{Participants}

Participants were chosen from those in the previous study who indicated a moderate to heavy habit of playing videogames. Specifically, we approached those participants who played at least three times a week, though not all agreed to take part in this further study. This gave 10 men and 1 women whose ages ranged from 16 to 22 and were all students but over a range of disciplines including history, medicine and chemistry.

\subsection{Materials and measures}

Addiction/engagement scores were already available for these participants as they had been selected from the previous study. Addiction/engagement was scored by adding up the scores on the 28 questions that are deemed to constitute factor 1 , as shown in Table 1. Scores for individual questions were reversed when the sign of the factor loading was negative. Inspection of the scores for all of the participants of study 1 suggested that the addiction/engagement scores were normally distributed.

Immersion was measured using a questionnaire that we have been developing for sometime and which was recently validated in a large online study [16]. The questionnaire probes for sense of control, loss of time, awareness of surroundings and related things. The only overlap between the immersion questionnaire and Charlton's CAAS is in asking whether participants experienced a sense of challenge. In some sense, the CAAS asks about the external characteristics of the player that indicate addiction/engagement whereas the immersion questionnaire is asking about internal states of the player specific to an instance of playing. The immersion questionnaire has also been seen to produce normally distributed scores in all previous studies.

As this was a diary study, it was expected that people would fill out one such questionnaire for each playing event. Thus, the 
degree of immersion experienced by the players was the average immersion score for each questionnaire filled out.

As both scales seem to be normally distributed, correlation was measured using Pearson's r.

\subsection{Procedure}

Participants were given several copies of the immersion questionnaire together with a short questionnaire on the playing session itself. They were then asked to fill out one copy of these questionnaires whenever they played videogames over a two week period. They were told that they could ask for more questionnaires at any time during the two weeks if they needed them.

\subsection{Results}

The 11 participants returned a mean of 3 questionnaires (mode of 2) with a maximum of 6 returns and a minimum of 1 . This may seem a low return rate over two weeks for players rated as heavy players but this may be because the participants were all students and the diary was taken during term time. The diary may also be incomplete.

The players played on average between 1 and 2 hours in their sessions with the shortest session being half and hour and the longest 8 hours. Games played were very varied and included Baldurs Gate, Cricket Captain, The Legend of Zelda: Twilight Princess and Warhammer 40,000. A variety of platforms were also used including PC, Xbox 360, Playstation, Nintendo DS and Nintend Wii. This summary supports our claim that we would be unable to provide a realistic gameplaying experience in a lab setting.

Table 2. Summary statistics for average immersion and engagement.

\begin{tabular}{|l|r|r|r|l|}
\hline Scale & Mean & \multicolumn{1}{l|}{ SD } & Min & Max \\
\hline Average immersion & 96.0 & 13.4 & 73.5 & 118.4 \\
\hline $\begin{array}{l}\text { Addiction/engagemen } \\
\mathrm{t}\end{array}$ & 86.1 & 15.1 & 67.0 & 108.0 \\
\hline
\end{tabular}

As there were varying numbers of immersion questionnaires returned for each participant, the mean immersion score of all playing sessions was used as the immersion score for each individual. Thus, the immersion score analysed indicates an average degree of immersion experienced by the participants. The immersion and addiction/engagement scores are summarized in Table 2. They indicate a good variation between the players on both scales. This is important as high degree of homogeneity in one variable can give an artificial correlation due to the regression line being essentially flat.

The correlation between average immersion and addiction/engagement was $\mathrm{r}=0.763, \mathrm{p}<0.01$. Moreover this is a substantial effect as the regression coefficient of engagement for predicting immersion is 0.68 (95\% confidence interval being 0.25 to 1.11 ), that is for every 10 point change in addiction/engagement score the immersion score changes by 6.8 points (or between 2.5 and 10.1 with $95 \%$ confidence). The scatterplot of immersion against engagement/addiction together with the regression line is shown in Figure 1.

It is also worth noting that there was no correlation between the total amount of time played or the number of instances people played and their immersion and engagement/addiction scores.

\subsection{Discussion}

As predicted, immersion does correlate with engagement/addiction, though, of course, correlation is no indicator of causation. Thus, it might be that because of player disposition to be highly engaged with videogames, the playing experience is more intensely immersive or that, because of more immersive playing experience, the player is more likely to become highly engaged. In practice, it seems likely to be a mix of the two with rewarding immersive experiences leading to better engagement and increased desire to play and longer playing times leading to an increased sense of immersion being lost for longer in the game.

What is notable in the data is the range of both immersion and engagement scores. As each question in both the immersion questionnaire and the adapted CAAS is scored from 1 to 5 , the maximum (minimum) possible scores are 160 (32) and 140 (28), respectively. Even though these individuals were selected because they played a lot, their scores varied as a substantial proportion of the full range. This suggests that immersion may not be the only reason why people play - we did not ask about motivations for playing. This makes it particularly interesting that immersion is in such a strong relationship to engagement/addiction: whereas there may be other reasons for playing, it is how immersive the experience is that is a good predictor for the tendency to high engagement and addiction.

\section{GENERAL DISCUSSION AND FURTHER WORK}

Overall, it seems that the immersive experience of playing games is strongly related to how highly engaged/addicted people are to playing videogames. What is perhaps somewhat frustrating in this work is that, if addiction and engagement had remained as separate factors as in Charlton's studies, it would be clearer whether immersion was important for addiction per se or simply for higher engagement.

To help interpret these findings then, it is worth dwelling more on the differences between immersion and high engagement. As already discussed, immersion is tapping into the subjective experience of players in relation to specific occasions on which they played. CAAS however asks about people's attitudes and behaviours outside of particular playing experiences. Thus, immersion could be viewed as in internal measure, or psychological measure, of engagement in playing videogames and the engagement/addiction factor as an external measure, or behavioural measure (albeit measured by self-report). Viewed in this way, it is perhaps not too surprising the immersion correlates to high engagement: they are the flip sides of the same coin. In this view, high engagement (external measure) occurs whenever immersion (internal measure) is strong.

This view however does not account for the incorporation into the high engagement factor of Brown's criteria for behavioural addiction. It is not only that highly engaged people want to and do play a lot, they also do so to detriment of themselves (missing sleep and meals) and their relationships with others (arguments with other, interfering with work). All but one of these criteria comes out clearly as components of high engagement. This suggests that in the domain of videogames, high engagement really does have much in common with behavioural addiction. With this fuller picture of high engagement, it seems that whilst immersion is viewed as an important and valued aspect of videogames, it is this very aspect that is involved in their addictiveness. 
Fortunately, videogames do not seem to be the destructive and socially detrimental force that other addictions are. Certainly in some extreme cases, the addiction is as bad as any other ruining relationships and destroying lives but on the whole the addicts, and specifically, the highly engaged players in our studies, were able to hold together functional lives and engage in normal social relations.

So whilst it may seem that videogame engagement is bad because it leads to addiction, there may be a more hopeful picture. Addiction to videogames could be just a form of high engagement, perhaps no worse than reading a really gripping book and then wanting to read a lot more. Indeed, both authors could claim to have lost sleep and missed meals through reading good books but we would also both claim to have our reading habits firmly under control and integrated into our social and functional lives.

Resolving these two contrasting interpretations seems like a good direction for future research. Using the adapted CAAS, we can rate people on their degree of engagement/addiction and see to what extent they are able to sustain a stable and functional life. In addition, it would be useful to see if the CAAS is actually measuring an underlying personality trait by placing it in relation to other personality measures that might indicate a propensity for high engagement such as Tellegen's Absorption scale [21]

It would also be useful to not only better identify people more likely to become addicted but games that are more addictive. It would be unethical to get people to play to the point that they are addicted but this research suggests that, using immersion, it would be possible to see which games give higher immersion and hence lead people down the route of high engagement and addiction. This however turns the usage of the immersion questionnaire on its side from measuring people playing games to measuring games played by people. This new use ought to be validated to ensure that the immersiveness of a game is a meaningful measure.

This study also helps to clarify that immersion is an important concept worth studying in its own right. Not only is it a widely used term of reference in videogaming culture but it has a real and measurable impact on people's relationships to videogames and to each other. Whilst game designers may wish to produce games that are more and more immersive, this research suggests that there may be ethical problems in relation to addiction. Only further study will reveal the full picture.

\section{ACKNOWLEDGMENTS}

Many thanks to Charlene Jennett for her assistance in framing the concept of immersion and Eduardo Calvillo and Sarah Faisal for their very helpful comments on this paper.

\section{REFERENCES}

[1] Agarwal, R., \& Karahana, E. (2000). Time flies when you're having fun: Cognitive Absorption and beliefs about information technology usage. MIS Quarterly, 24 (4), 665-694.

[2] American Psychiatric Association (2000). DSM-IV-TR: Diagnostic and Statistical Manual of Mental Disorders. Arlington, VA: American Psychiatric Association Press.

[3] BBC News (2005). S Korean dies after games session. http://news.bbc.co.uk/1/hi/technology/4137782.stm

[4] BBC News (2006). Videogame addiction clinic opens. news.bbc.co.uk/1/hi/technology/5191678.stm
[5] Brown, E., \& Cairns, P. (2004). A grounded investigation of game immersion. ACM CHI 2004, New York: ACM Press, 1279-1300.

[6] Brown, R.I.F. (1991). Gaming, gambling and other addictive play. In Kerr, J.H. \& Apter, M.J. (eds) Adult play: a reversal theory approach. Amsterdam: Swets \& Zeitlinger, 101-118.

[7] Carr, D. (2006) Play and pleasure. In Carr,.D., Buckingham, D., Burn, A. \& Schott, G. (eds) Computer games: text, narrative and play. Cambridge: Polity Press, pp.45-48.

[8] Chappell, D, Eatough, V, Davies, M.N.O \& Griffiths, M (2006). EverQuest - it's just a computer game, right? An interpretative phenomenological analysis of online gaming addiction. Int, J. of Mental Health Addiction, 4, 205-216.

[9] Charlton, J.P. (2002). A factor-analytic investigation of computer 'addiction' and engagement. British J. of Psychology, 93,329-344.

[10] Charlton, J.P. \& Birkett, P.E. (1995). The development and validation of the computer apathy and anxiety scale. $J$. of Educational Computing Research, 21, 393-412.

[11] Charlton, J.P., \& Danforth, I.D.W. (2007) Distinguishing addiction and high engagement in the context of online game playing. Computers in Human Behaviour, 23, 15311548.

[12] Chen, V.H. \& Duh, H.B-L (2007) Understanding social interaction in World of Warcraft. Proc. of Int. Conf. on Advances in Computer Entertainment Technology, New York: ACM Press, 21-24.

[13] Csikszentmihalyi, M. (1990). Flow: The Psychology of Optimal Experience. New York: Harper and Row.

[14] Duchenaut, N., Yee, N., Nickel, E., Moore, R.J. (2006). "Alone together?" Exploring the social dynamics of massively multiplayer online games. ACM CHI 2006, New York: ACM Press, 407-416.

[15] Jayisgames.com (2007) Westward. jayisgames.com/archives/2006/12/westward.php

[16] Jennett, C.I., Cox, A.L., Cairns, P., Dhoparee, S., Epps, A., Tijs, T. \& Walton, A. (under revision). Measuring and defining the experience of immersion in games. Accepted in Int. J. of Human-Computer Studies.

[17] Kline, P. (1994). An Easy Guide to Factor Analysis. New York: Routledge.

[18] Rockstar Games (2007). Grand Theft Auto IV. $\mathrm{http}: / /$ www.rockstargames.com/IV

[19] Slater, M., Usoh, M. \& Steed, A. (1994). Depth of presence in virtual environments. Presence: Teleoperators and Virtual Environments, 3 (2), 130-140.

[20] Sweetser, P. \& Wyeth, P. (2005). GameFlow: a model for evaluating player enjoyment in games. ACM Computers in Entertainment, 3(3), 1-24.

[21] Tellegen, A. \& Atkinson, G. (1974). Openness to absorbing and self-altering experiences ("absorption"), a trait related to hypnotic susceptibility. Journal of Abnormal Psychology, 83, 268-277.

[22] Turbine, Inc (2007). Asheron's Call. http://ac.turbine.com/

[23] Ubisoft, inc (2007). Myst IV. http://www.mystworlds.com/ 
From Immersion to Addiction in Videogames

[24] Weinberg, G.M. (1971) The psychology of computer programming. New York: Van Nostrond Reinhold.

[25] Witmer, B. G. \& Singer, M. J. (1998). Measuring presence in virtual environments: A presence questionnaire. Presence: Teleoperators and Virtual Environments, 7 (3), 225-240 